\title{
Recursos renovables en el mercado eléctrico español: Instrumentos y efectos*
}

\author{
Aitor Ciarreta-Antuñano \\ Universidad del País Vasco \\ Carlos Gutiérrez-Hita \\ Universitas Miguel Hernández
}

\section{Resumen}

La implantación de la directiva 2001/77/CE relativa a la promoción de electricidad generada a partir de fuentes renovables fijó la cuota del 22,1\% sobre el consumo de electricidad de la UE en 2010. Además establecía diferentes mecanismos que los países miembros podían adoptar según sus necesidades. En este trabajo se analizan dichas mecanismos, su implementación en España a través del Real Decreto 661/2007 y el grado de cumplimiento de los objetivos planteados. Se concluye con un análisis de la efectividad de las medidas y recomendaciones sobre el diseño del mercado.

Palabras Clave: Recursos renovables, mercado eléctrico, marco regulatorio.

Clasificación JEL: Q2, Q4.

\begin{abstract}
The implementation of the Directive 2001/77/EC on the promotion of electricity produced from renewable sources set the target of $22.1 \%$ of electricity consumption in the EU in 2010. Moreover, various mechanisms were established that member countries could adopt depending on their needs. This paper analyzes these mechanisms, their implementation in Spain by mean of Real Decreto 661/2007 and the degree of fulfilment of objectives. It concludes with an analysis of the effectiveness of measures and recommendations on market design.
\end{abstract}

Keywords: Renewable resources, electricity market, regulatory framework.

JEL Classification: $Q 2, Q 4$.

“... yo quisiera gritar que ese tal oro negro no es más que un negro veneno,..."

El progreso (ROBERTO CARLOS)

* Los autores agradecen el apoyo financiero del Ministerio de Ciencia y Tecnología a través de los proyectos ECO2009-09120 y MTM2008-06778-C02-01/MTM y al Gobierno Vasco a través del proyecto DEUI, IT-313.07. Los autores agradecen también los comentarios y puntualizaciones realizadas por un evaluador anónimo. 


\section{Introducción}

En el actual contexto de cambio de modelo económico, de incorporación de países económicamente emergentes al consumo de energía a gran escala y de alteración del clima (como consecuencia de la actividad productiva a escala industrial desde el siglo XVIII, fundamentalmente) los procesos de generación de energía en general y la eléctrica en particular (que está llamada a ser el modo de energía más utilizada en el futuro próximo), necesitan apoyarse en la utilización generalizada de fuentes de energía renovables (FER, en adelante) ${ }^{1}$. Sin embargo, el uso y explotación de los diferentes tipos de fuentes de energía no renovable, en particular los recursos fósiles, seguirá creciendo en valores absolutos aunque su ponderación en el mix tecnológico de producción decrezca. La utilización como inputs energéticos de los recursos fósiles además de los renovables depende, fundamentalmente, de los siguientes factores:

i) el estado de la técnica y la incorporación de los nuevos procesos de producción basados en FER generan niveles de energía inferiores al total de la demanda energética y de los incrementos de ésta;

ii) las fuentes de energía no renovable generan costes sociales y medioambientales que no están debidamente internalizados mediante sistemas de penalización (vía impuestos o tasas);

iii) por último, las fuentes de energía no renovable continúan estando apoyadas, en muchos casos, por lobbies políticos y económicos que dificultan la incorporación de las FER a los procesos productivos. Su inclusión podría suponer una pérdida de poder de dichos lobbies a ambos niveles, político y económico.

En primer lugar, los recursos renovables se encuentran en un proceso de incorporación a los procesos productivos, por lo que aún no se puede evaluar su eficiencia en términos económicos. Por una parte, su estado de desarrollo depende en gran medida de los avances técnicos. Por ello están, en este sentido, en desventaja con los procesos de generación de energía que utilizan combustibles fósiles, los cuales están amortizados y su eficacia energética suficientemente probada a lo largo de las últimas décadas. Por otra parte, un problema añadido de las FER es su necesaria modularidad o complementariedad para ser utilizados como inputs a gran escala en el mix tecnológico eléctrico. En efecto, las FER carecen de la continuidad de generación de que gozan los procesos con combustibles fósiles. Aquellas son intermitentes en su naturaleza, debido a que van ligadas a factores climáticos o difícilmente predecibles al 100\% (este es el caso de la energía eólica y solar fotovoltaica). Así pues, constituye una cuestión abierta y aun no solucionada el poder disponer de energía eléctri-

${ }^{1}$ En el artículo, la expresión "recursos renovables" y el acrónimo FER son utilizados indistintamente. 
ca generada a partir de FER sin incurrir en el riesgo de interrupciones de suministro al sistema provocando que la oferta no satisfaga la demanda. En este sentido, parece razonable que la producción de energía eléctrica se apoye en distintas fuentes, para minimizar el impacto que la carencia de alguna de ellas tenga en el sistema.

En segundo lugar, a consecuencia de los usos y transformaciones de la energía generada con recursos no renovables se generan externalidades negativas, tanto a nivel local como a nivel global, con efectos de distinta magnitud en el medio y largo plazo. Así, las emisiones de partículas procedentes de centrales eléctricas causan un impacto local, mientras que las emisiones de $\mathrm{CO}_{2}$ causan un impacto global; los derrames de crudo en las refinerías suelen tener un impacto limitado en el tiempo, mientras que el problema de cómo gestionar los residuos radiactivos constituye un problema más a largo plazo. Las citadas externalidades no son, sin embargo, internalizadas mediante la aplicación de impuestos y/o tasas a la energía generada mediante recursos fósiles ${ }^{2}$. Este hecho genera que los costes sociales no se igualen al coste de oportunidad que tiene la generación de electricidad mediante estos recursos. A este respecto, la disposición de la Directiva Europea 2001/77/CE sobre la garantía de origen asegura una identificación de la procedencia de la electricidad y que el usuario final pueda asumir parte del gravamen aplicado a la electricidad generada con combustibles fósiles ${ }^{3}$.

Por último, un problema añadido es la existencia de lobbies económicos que preservan el suficiente poder político para mantener altas cuotas de recursos energéticos fósiles en la generación eléctrica, manteniendo así a empresas en posiciones de privilegio con el fin de ejercer poder de mercado (y en ocasiones abuso de posición de dominio en el sentido del art. 81 y 82 del Tratado de la Unión). Cabe mencionar también que la intervención estatal puede ayudar o dificultar procesos de asociación o fusión entre empresas con el ánimo de salvaguardar los intereses nacionales. Sin embargo, dichas intervenciones son, en muchas ocasiones, difícilmente defendibles desde un punto de vista de eficiencia energética.

En suma, la preocupación por el cambio climático, la calidad del aire y la seguridad en la producción de energía han aumentado el interés en el potencial de las FER para desplazar a los recursos energéticos no renovables y potencialmente peligrosos para la salud humana y el medio ambiente. Sin embargo, la falta de un adecuado sistema de incentivos y penalizaciones a la producción de energía con combustible fósil y la existencia de intereses políticos y económicos pueden dificultar, y de hecho lo hacen, una adecuada combinación tecnológica que responda a un modelo energético sostenible y eficiente.

\footnotetext{
${ }^{2}$ En este sentido, no todas las externalidades causadas por los recursos fósiles se pueden internalizar mediante estos mecanismos. En un contexto dinámico, sería deseable diseñar un sistema de incentivos y penalizaciones para asegurar una provisión energética no sólo eficiente desde el punto de vista económico sino también con el objetivo de preservar el medioambiente, la biodiversidad y la igualdad en el acceso a la misma en el medio y largo plazo.

${ }^{3}$ Este último aspecto constituye una cuestión abierta al depender de las preferencias individuales de los consumidores y consideraciones más generales de gestión de la demanda.
} 
En este artículo revisamos las principales medidas de promoción de las FER adoptadas en el proceso de liberalización del mercado eléctrico español. En primer lugar, repasamos los cambios normativos y regulatorios en el marco de la Unión Europea (UE, en lo sucesivo) y su transposición al mercado interno español. En segundo lugar, describimos los instrumentos utilizados en el mercado eléctrico español y en particular la aplicación del RD 661/2007 y su influencia en los resultados del mercado. Por último, evaluamos la efectividad de dichas medidas y se enumeran una serie de recomendaciones.

\section{El marco regulatorio y las FER en el mercado eléctrico}

En esta sección se analizan los principales cambios acontecidos en la normativa europea y española en relación a la promoción y uso de las FER en el contexto de liberalización del mercado eléctrico ${ }^{4}$.

\subsection{Normativa y acciones de la $U E$}

La liberalización del sector eléctrico junto con la reducción de las emisiones de gases de efecto invernadero son dos objetivos principales de la política energética de la UE. Las políticas de energía renovable se iniciaron en la UE con el Plan de investigación, desarrollo y demostración de 1974. Sin embargo, la implementación de políticas dirigidas comenzó en 1994, cuando en la Conferencia de Madrid se sentaron las bases de las primeras políticas orientadas a potenciar las FER. La Directiva Comunitaria 1996/92/CE, además de establecer las normas comunes para la liberalización del mercado interior de la electricidad, tenía como objetivo la garantía del suministro respetando el medio ambiente. Un año más tarde, en noviembre de 1997, la Comisión Europea emitió el comunicado Energía para el futuro: fuentes de energía renovables - Libro Blanco para una estrategia y un plan de acción comunitarios (E-FER), en el que se propuso doblar la cuota de participación de las fuentes de energía renovable en el consumo interior bruto de energía dentro de la UE. En el marco del Protocolo de Kioto, adoptado ese mismo año, la UE se comprometió a reducir sus emisiones de gases de efecto invernadero en un $8 \%$ entre 2008 y 2012 respecto de los niveles de 1990. Asimismo, la Comisión Europea en su Libro Verde de noviembre de 2000 "Hacia una estrategia europea de seguridad del abastecimiento energético", proponía el fomento de las energías renovables y de la cogeneración como energías necesarias para lograr el doble objetivo de reducir la dependencia energética y de limitar la emisión de gases de efecto invernadero 5 .

${ }^{4}$ Esta sección está inspirada y toma varios elementos de Ciarreta y Gutiérrez Hita (2009).

${ }^{5}$ Consultar también Jamasb et al. (2003). 
La Directiva 2001/77/CE relativa a la promoción de la electricidad generada a partir de FER en el mercado interior de la electricidad, fijó la cuota del 22,1\% de electricidad generada a partir de FER sobre el consumo de electricidad de la UE en el año $2010^{6}$. Este objetivo del $22,1 \%$ a nivel comunitario se traduce en el caso de España en un 29,4\%. Por otra parte, la Directiva 2003/87/CE (que modifica la Directiva 96/61/CE) del Parlamento Europeo y del Consejo por la que se establece un régimen para el comercio de derechos de emisión de gases de efecto invernadero en la Comunidad, establecía un régimen de comercio de derechos de emisión de gases de efecto invernadero con vistas a reducir dichas emisiones en la UE de forma eficiente.

El 9 de marzo de 2007 el Consejo Europeo de Primavera, con el apoyo del Parlamento Europeo y de los Estados miembros, acordó establecer como objetivo vinculante que el $20 \%$ de su consumo energético de 2020 proceda de fuentes renovables, teniendo en cuenta la situación específica de cada Estado miembro. Así, desde el 2007 el compromiso 20/20/20 forma parte del llamado "paquete verde" que se refiere a un triple objetivo para el año 2020 por el cual se debe conseguir que se emita a la atmósfera un $20 \%$ menos de $\mathrm{CO}_{2}$ que en 1990, se consuma un $20 \%$ menos de energía en términos relativos y se genere el $20 \%$ de energía primaria con FER.

Los principales mecanismos regulatorios dentro de la UE son instrumentos financieros e incentivos fiscales. Los instrumentos financieros son incentivos económicos que estimulan la transformación tecnológica de actividades con un reducido impacto ambiental. Las más destacadas son los sistemas basados en primas, ayudas a la inversión y exenciones o reducciones fiscales. En un sistema basado en primas los generadores que utilizan FER reciben una retribución extra por cada Kw/h suministrado. Hay esencialmente dos categorías de mecanismos de apoyo directo mediante primas dentro de la UE: los sistemas basados en cuotas, y los sistemas de precio fijo. Bajo el sistema de cuotas, los productores están obligados a producir una cuota fija de energía mediante FER, determinada a través de un mecanismo de competencia. Dos mecanismos diferentes operan en la actualidad: los certificados ecológicos y planes de licitación. El sistema de precios fijos implica que ninguna cuota o límite máximo sea fijado para las FER. Por último, los incentivos fiscales incluyen subvenciones o deducciones fiscales para fomentar el desarrollo tecnológico de algunas tecnologías cuyo coste es excesivamente elevado para ser llevado a cabo exclusivamente mediante iniciativa privada.

\subsection{Transposición de la normativa europea en España}

La normativa española incluye la generación de electricidad mediante recursos renovables dentro del llamado régimen especial, que incluye además la cogeneración y los residuos. El régimen especial se reguló en España en 1980 mediante la

${ }^{6}$ Con la ampliación de la UE hacia la Europa del Este en 2004 el objetivo global se redujo al $21 \%$. 
Ley 82/1980 de Conservación de la Energía, estableciéndose los objetivos de mejorar la eficiencia energética de la industria y de reducir la dependencia del exterior. En la década de los noventa, el Plan Energético Nacional 1991-2000 estableció incentivos para la cogeneración y la producción de energía con recursos renovables. Así, la Ley 40/1994 de Ordenación del sistema eléctrico nacional (LOSEN) dejó consolidado el concepto de régimen especial como tal, publicándose el Real Decreto 2366/1994 sobre producción de energía eléctrica por instalaciones hidráulicas, de cogeneración y otras abastecidas por FER.

La promulgación de la Ley 54/1997 del sector eléctrico, que marca el proceso de liberalización del mercado, introdujo la competencia en la generación mientras que la transmisión y la distribución permanecieron reguladas. La ley tenía por objeto conciliar la liberalización del sistema eléctrico con el objetivo de garantizar el suministro al menor precio posible y minimizando el impacto ambiental. Se diferenció a los productores de energía eléctrica en régimen ordinario de aquellos con instalaciones acogidas al régimen especial, que debían tener una potencia instalada menor o igual a $50 \mathrm{MW}^{7}$. Se establecía para estas últimas la posibilidad de incorporar su energía excedentaria al sistema a tarifa regulada (cediendo la energía directamente a un distribuidor) o participar directamente en el mercado de producción (directamente o a través de un representante) $)^{8}$. Además, se concedió prioridad a gestionar la electricidad generada por FER a través de la red en vez de introducirla directamente del productor a la red de distribución de media y baja tensión (transposición de la Directiva Europea 1996/92/CE). La CNE gestiona el Sistema de Garantía de Origen y Etiquetado de la electricidad desde el 1 de diciembre 2007 para garantizar el origen de la energía que se consume y el impacto ambiental asociado adaptándose así a la normativa europea ${ }^{9}$.

El Plan de Fomento de Energías Renovables (PFER) aprobado por el Gobierno el 30 de diciembre de 1999 estableció los objetivos de crecimiento necesarios en cada una de las tecnologías consideradas como renovables, para conseguir que la producción con estas fuentes representase el $12 \%$ del consumo español de energía primaria en el año 2010. Sin embargo, dada la nula participación de las instalaciones de régimen especial en el mercado bajo la aplicación del Real Decreto 2818/1998, el

${ }^{7}$ El Libro Blanco de 1997 promovió un programa para estimular el uso de FER. Este consistía básicamente en la aplicación de diferentes instrumentos políticos, siendo el más relevante el programa de "ayudas para la generación eléctrica mediante FER y ciclos combinados", que proporciona incentivos para nueva capacidad instalada de plantas que utilizan FER. Además, el Real Decreto 2818/1998 estableció la regulación concreta de la retribución de la energía vertida en régimen especial, ajustándose a lo indicado en la Ley 54/97.

${ }^{8}$ En ambos casos la empresa distribuidora debía gestionar la facturación y abonar al productor los importes de las tarifas, primas y complementos. A partir del 1 de noviembre de 2009 la CNE determina estos conceptos. Además, estos importes deben ser sometidos a lo establecido en el Real Decreto 2017/1997.

${ }^{9}$ La Directiva Europea 2003/54/CE relativa al mercado eléctrico interno, establece en el artículo tercero la obligatoriedad de aportación de información por parte de los productores de electricidad del origen y el impacto ambiental de la energía producida. 
Real Decreto-Ley 6/2000 de medidas urgentes de intensificación de la competencia en mercados de bienes y servicios, estableció la obligación para las instalaciones del Real Decreto 2366/1994 con una potencia superior a 50 MW de participar en el mercado de producción.

Con el Real Decreto 661/2007 se establece un nuevo marco regulatorio para el régimen especial. El titular de la instalación puede optar por vender su excedente de energía eléctrica producida a un distribuidor, percibiendo por ello una retribución en forma de tarifa regulada y única para todos los períodos de programación, que se define como un porcentaje de la Tarifa Eléctrica Media o de Referencia (TMR, en lo sucesivo) de cada año; o bien por vender dicho excedente directamente a través del mercado diario o mediante un contrato bilateral, percibiendo en este caso el precio negociado en el mercado, un incentivo por participar en él y una prima (si la instalación concreta tenía derecho a percibirla). En éste sentido, la diferencia fundamental con el Real Decreto 436/2004 es que toda la producción acogida al régimen especial a tarifa debe presentar ofertas en el mercado de producción a precio cero por medio de un representante ${ }^{10}$. Este incentivo y esta prima complementaria se definen también genéricamente como un porcentaje de la TMR. La revisión de las tarifas, primas e incentivos se realizaría cada 4 años a partir de 2006, y sólo afectaría a las nuevas instalaciones ${ }^{11}$. Otros cambios significativos que este Real Decreto plantea frente a la regulación anterior son los siguientes:

- La retribución del régimen especial deja de estar unida a la TMR estando la actualización de las tarifas, primas y complementos ligada a la evolución de diversas variables micro y macroeconómicas relacionadas con el mercado.

- La retribución a cada tipo de FER no es homogénea y depende tanto del volumen producido como del horizonte temporal de explotación de cada planta. Así por ejemplo, las instalaciones encuadradas en el grupo b.1.1 (solar fotovoltaica) perciben una prima diferente si la potencia es menos de $100 \mathrm{~kW}$, entre $100 \mathrm{~kW}$ y $10 \mathrm{MW}$ y entre $10 \mathrm{MW}$ y $50 \mathrm{MW}^{12}$.

- Se establece una prima de referencia y unos límites superior e inferior para la generación procedente de renovables que participa en el mercado.

- Los nuevos parques eólicos deberán ser capaces de mantenerse conectados a la red ante una breve caída de tensión en la misma.

- Se permite la hibridación en instalaciones de biomasa y solar termoeléctrica.

${ }^{10}$ En el régimen especial a tarifa, la distribuidora es su representante para la participación en el mercado, cobrando éstos al régimen especial por este servicio un cargo de $0,5 \mathrm{c} € / \mathrm{kWh}$.

${ }^{11}$ Se derogan el Real Decreto 841/2002 y el Real Decreto 2818/1998 y se establece un período transitorio para las instalaciones acogidas al Real Decreto 2366/1994 (DT1 ${ }^{a}$ Real Decreto 436/2004) y al Real Decreto 2818/1998 (DT2 ${ }^{\mathrm{a}}$ Real Decreto 436/2004), que tienen la opción de mantenerse en el antiguo régimen económico que les corresponde.

${ }_{12}$ Para una completa información de las primas percibidas por cada FER y la división en grupos y subgrupos ver el Capítulo 1 (art. 2) y Capítulo IV, sección $3^{\text {a }}$ (art. 36 al 41). 
Con la publicación de la Ley 17/2007 (que modifica la Ley 54/1997 del sector eléctrico) para adaptarla a lo dispuesto en la Directiva 2003/54/CE sobre normas comunes para el mercado interior de la electricidad, se acuerda modificar el PFER para adecuarlo al objetivo 20/20/20 antes mencionado. Posteriormente, ha sido aprobado el Real Decreto 1578/2008 de retribución de la actividad de producción de energía eléctrica mediante tecnología solar fotovoltaica para las instalaciones posteriores a la fecha límite de mantenimiento de la retribución del Real Decreto 661/2007 para la citada tecnología. Se clasifican las nuevas instalaciones en dos tipologías, según estén ubicadas en cubiertas (tipo I) o en suelo (tipo II).

Por último, el Real Decreto Ley 6/2009 establece el mecanismo de registro de preasignación de retribución para las instalaciones del régimen especial, con el que se pretende controlar el cumplimiento de los objetivos de planificación. Las tarifas y primas vigentes para las FER y residuos se encuentran establecidas en la Orden ITC/3801/2008.

\section{Instrumentos y políticas de fomento para la utilización de FER}

La promoción de las fuentes renovables deberá llevar en el medio plazo a que los sistemas de generación de electricidad se basen en mayor medida que en la actualidad en recursos renovables. Pero el aumento de los recursos renovables en la combinación tecnológica para la producción de electricidad requiere un apoyo firme y eficiente de políticas de promoción y una apropiada regulación del mercado eléctrico.

El fallo de mercado provocado por la externalidad negativa que generan los combustibles fósiles y la externalidad positiva generada por la utilización de FER puede ser solucionada por la regulación de las emisiones de $\mathrm{CO}_{2}$ y el apoyo a la generación de FER. Si el daño medioambiental pudiera ser estimado, el problema se resuelve mediante la introducción de un impuesto Pigouviano, restableciendo el equilibrio competitivo entre ambos tipos de tecnologías. Sin embargo, los problemas inherentes a la introducción de tales impuestos (principalmente políticos) llevan a que el coste medioambiental del uso de combustibles fósiles se refleje solo parcialmente en los precios de mercado ${ }^{13}$. Así, el apoyo brindado a las FER está justificado desde el punto de vista de una compensación por evitar las externalidades negativas. A continuación vamos analizar la eficiencia relativa de los dos instrumentos clásicos utilizados para difundir y potenciar el uso de recursos renovables en la generación eléctrica y la evolución reciente del uso de FER en el sistema eléctrico español. En particular,

i) Instrumentos basados en precios o en cantidades.

ii) Certificados verdes: documentos otorgados por la Autoridad Regulatoria Nacional a los generadores de electricidad con FER según el importe y la

${ }^{13}$ De hecho, sólo Holanda y Dinamarca han impuesto tasas a las emisiones de $\mathrm{CO}_{2}$. 
calidad medioambiental de la electricidad así producida. Mediante este sistema se impone a las compañías distribuidoras de electricidad la obligación de que un determinado porcentaje de su suministro provenga de FER.

Las políticas de los estados miembros de la UE y en particular en España para promover las FER muestran que la elección de los instrumentos está basada en la eficiencia. La noción de eficiencia implica que la competencia entre diferentes tecnologías sea tal que cada una realice una contribución óptima al sistema dependiendo de su coste marginal de generación. Las medidas de apoyo asignadas a cada tecnología deben estar, así pues, en consonancia con su función de costes. Por un lado, deben incentivar la producción al mínimo coste posible en cada período a través de las tecnologías disponibles: la eficiencia estática. Por otro, deben existir incentivos permanentes para conseguir reducciones de costes a través del progreso tecnológico, de modo que en última instancia se alcance la plena competencia entre tecnologías: la eficiencia dinámica.

Aparte de las cuestiones relacionadas con la eficiencia, hay otras barreras relacionadas con características técnicas y económicas de las FER para potenciar su difusión. En particular, son procesos especialmente intensivos en capital y, aún más importante, en ocasiones son incapaces de generar energía mediante procesos continuos. La primera característica tiene que ver con el aspecto ya comentado del apoyo mediante primas y subvenciones a las FER y tasas a los recursos contaminantes. El segundo aspecto tiene que ver con su modularidad, y es una cuestión dinámica que deber ser resuelta mediante dos vías,

i) el progreso tecnológico y la incorporación de los mismos a los procesos de generación de electricidad mediante FER; y,

ii) mejoras en los procesos de transporte de la electricidad y de distribución mediante una mejora del funcionamiento del mercado y el proceso de liberalización.

Existen básicamente tres tipos de instrumentos para introducir las FER en la generación eléctrica: los sistemas basados en precios, los sistemas basados en cantidades y, más recientemente, una variación de éstos últimos, los certificados verdes. Su elección constituye un debate abierto y depende de varias cuestiones relacionadas con el desarrollo del mercado, el proceso liberalizador y el estado de la tecnología.

- El sistema de primas. Es el sistema más antiguo y más profusamente usado (aparte de España, también en Dinamarca, Alemania e Italia). Mediante este sistema los distribuidores están obligados a adquirir en primer lugar la energía producida por las FER a un precio determinado por el regulador durante un período de tiempo determinado. Desde el punto de vista del generador opera como un subsidio.

- El sistema de pujas competitivas. Usado principalmente en el Reino Unido y Francia (en éste último estado hasta 2000). En este caso el regulador reserva una 
proporción del mercado a la producción mediante FER y se desarrolla la competencia entre los generadores que utilizan estos recursos. Los distribuidores están obligados a adquirir el total de la cantidad producida en esta parte del mercado. Las ofertas de producción son clasificadas en orden creciente de precios hasta que la cantidad propuesta se alcanza.

- Certificados verdes. Es un instrumento similar a un sistema basado en cantidades pero difiere en que cada operador se le asigna un objetivo cuantitativo. Los certificados verdes tienen por objetivo que la energía producida a partir de FER se pueda llegar a convertir en parte integral del mercado eléctrico. Su uso se debería generalizar a medida que las FER intervengan en mayor proporción en el mix tecnológico eléctrico y los condicionantes medioambientales (la necesidad de reducir el volumen de $\mathrm{CO}_{2}$ vertido a la atmósfera, una mayor concienciación pública sobre el uso de energías limpias...) hagan que el uso de recursos fósiles se reduzca drásticamente. Mediante este sistema se define una cuota fija de electricidad que se divide en la cantidad total de certificados, decidida por el Estado y repartida entre los generadores en base a sus costes para preservar una asignación eficiente. Así, los costes marginales son igualados entre generadores. Dichos certificados se asignan a los agentes del mercado para ser utilizarlos de dos maneras diferentes:

i) vendiendo la electricidad generada al precio de mercado a través del mercado diario al precio marginal fijado; o

ii) vendiendo los certificados a operadores que deben alcanzar una determinada cuota en el mercado secundario de certificados verdes.

Las transacciones de certificados verdes son especialmente interesantes en contextos internacionales donde las posibilidades de transacciones son mucho más altas que a nivel nacional, por ello es uno de los instrumentos prioritarios de las UE en el medio y largo plazo a medida que el mercado único europeo de electricidad se vaya desarrollando.

El actual sistema de primas en España debería poder ser reemplazo gradualmente en los próximos años por un sistema de certificados verdes si se dan ciertos condicionantes,

i) que el rápido crecimiento de la generación y el correspondiente incremento agregado de los subsidios a los productores que utilizan FER que son financiados con fondos públicos sea insostenible ${ }^{14}$;

ii) que la liberalización del mercado permita un mayor número de consumidores y un mayor volumen de consumo de electricidad, a la vez que la libertad de elegir el proveedor;

iii) que los procesos basados en FER se puedan equiparar en eficiencia a aquellos que utilizan combustibles fósiles.

${ }^{14}$ Hay que destacar que, aunque el subsidio total por toda la electricidad generada ha crecido, el pago recibido por MWh es decreciente en el tiempo. 
Entre las ventajas más significativas de los certificados verdes cabe destacar las siguientes,

i) estimulan nuevas formas de utilización de FER en generación. Los objetivos de política medioambiental pueden ser fácilmente definidos en términos cuantitativos, permitiendo evaluar el progreso en función de un punto de referencia conocido mediante la introducción de cuotas cada vez más exigentes.

ii) incentiva las reducciones de costes. La creación de certificados verdes y de un mercado secundario para su transacción da un doble incentivo para reducir costes. Primero, la electricidad producida por medio de FER puede ser vendida y transaccionada a través de la red al precio de mercado, que tiende a ser más bajo mediante la introducción de competencia. Segundo, los productores de energía a través de FER están bajo presión constante por medio de la competencia debido al mercado de certificados verdes, creando incentivos para potenciales inversores y a ser diligentes en la gestión de los costes tanto de nuevos proyectos como de las instalaciones ya operativas.

El interés teórico de los certificados no debe sin embargo enmascarar los problemas asociados con la organización de las transacciones. Para que funcione un mercado secundario de certificados verdes se debe garantizar la certificación de que la energía proviene de FER, abrir y gestionar registros de comercio, realizar auditorias y cumplimentar una contabilidad exhaustiva, además instaurar un sistema de multas por incumplimientos.

\section{El impacto de las FER en el mercado español}

La Directiva 2001/77/CE otorga libertad a cada Estado miembro para elegir los mecanismos de apoyo al uso de los recursos renovables. En la actualidad, el marco legal para el régimen especial se articula entorno al RD 661/2007 (que deroga el RD 436/2004) manteniendo los principios básicos de la normativa anterior e introduciendo cambios con el objetivo de cumplir la Directiva 2001/77/CE: al menos el 29,4\% del consumo total de electricidad deberá proceder de fuentes renovables en el año 2010. En España el instrumento básico de promoción de las FER son las primas. Hay dos posibilidades para vender la electricidad generada por fuentes renovables en el mercado eléctrico español:

- Los generadores pueden introducir directamente la electricidad en la red, sin necesidad de pasar a través del mercado diario, y obtener una tarifa regulada única para cada hora del día. Alternativamente, pueden vender a través del operador del mercado, aunque las ofertas se hacen a precio cero, a diferencia de las ofertas procedentes de otras tecnologías ${ }^{15}$.

${ }^{15}$ Una excepción es la energía generada por plantas nucleares que también es introducida a precio cero por motivos estratégicos. 
- Los generadores pueden hacer ofertas de electricidad en la subasta de precio uniforme del mercado diario o al precio fijado mediante la contratación bilateral, con un subsidio para compensar el incremento del costo de la generación en comparación con el precio de mercado.

La CNE resuelve el pago del régimen especial y publica un informe sobre las compras de energía que incluye la información más relevante sobre la actividad mencionada $^{16}$. En el Cuadro 1 se resume la evolución desde 2002 hasta 2008 de la electricidad transaccionada en el mercado por empresas y el porcentaje del régimen especial sobre el total.

\section{CUADRO 1}

\section{CUOTAS DE MERCADO POR EMPRESA Y CUOTA DEL RÉGIMEN ESPECIAL}

\begin{tabular}{|c|c|c|c|c|c|c|c|c|c|}
\hline Año & EN & IB & UF & HC & VI & GN & Reg. Esp. & Otras \\
\hline 2002 & 0,35 & 0,25 & 0,11 & 0,06 & 0,04 & 0,01 & 0,15 & 0 \\
\hline 2003 & 0,32 & 0,24 & 0,10 & 0,05 & 0,02 & 0,02 & 0,14 & 0,11 \\
\hline 2004 & 0,31 & 0,21 & 0,09 & 0,05 & 0,02 & 0,02 & 0,14 & 0,16 \\
\hline 2005 & 0,30 & 0,18 & 0,10 & 0,05 & 0,03 & 0,03 & 0,10 & 0,21 \\
\hline 2006 & 0,26 & 0,18 & 0,10 & 0,04 & 0,02 & 0,07 & 0,03 & 0,30 \\
\hline 2007 & 0,26 & 0,18 & 0,11 & 0,04 & 0,02 & 0,06 & 0 & 0,33 \\
\hline 2008 & 0,25 & 0,18 & 0,10 & 0,03 & 0,02 & 0,06 & 0 & 0,36 \\
\hline \multicolumn{7}{|c|}{} & & DEMANDA & \\
\hline 2002 & 0,35 & 0,31 & 0,10 & 0,05 & 0,01 & 0 & 0,15 & 0,03 \\
\hline 2003 & 0,34 & 0,32 & 0,10 & 0,05 & 0,01 & 0,01 & 0,14 & 0,03 \\
\hline 2004 & 0,32 & 0,31 & 0,11 & 0,05 & 0,01 & 0,02 & 0,14 & 0,04 \\
\hline 2005 & 0,33 & 0,32 & 0,12 & 0,05 & 0,01 & 0,02 & 0,10 & 0,05 \\
\hline 2006 & 0,44 & 0,24 & 0,15 & 0,06 & 0,01 & 0,01 & 0,03 & 0,06 \\
\hline 2007 & 0,39 & 0,33 & 0,15 & 0,07 & 0,01 & 0,01 & 0 & 0,04 \\
\hline 2008 & 0,38 & 0,30 & 0,15 & 0,07 & 0,01 & 0,02 & 0 & 0,07 \\
\hline
\end{tabular}

Fuente: OMEL, CNE y construcción propia. Acrónimos: EN: Endesa, IB: Iberdrola, UF: Unión Fenosa, HC: Hidrocantábrico, VI: Riesgo, GN: Gas Natural.

${ }^{16}$ Con anterioridad a 2007, la publicación de los precios finales y las cuotas de productores y compradores de electricidad correspondía al operador del mercado, OMEL. 
Nótese que a medida que las medidas liberalizadoras han sido más efectivas, las dos grandes empresas, Endesa e Iberdrola, han perdido peso en el mercado, aunque siguen obteniendo un alto porcentaje del mismo en conjunto (tanto en generación como en distribución y comercialización). Asimismo, la evolución del régimen especial es negativa hasta el año 2007. A partir de entonces, toda la electricidad generada a través del régimen especial (y por ende aquella mediante FER) ha de ser introducida en el mercado diario ${ }^{17}$. No obstante, como veremos más adelante, el porcentaje de energía generada mediante FER ha crecido notablemente tanto en términos relativos como absolutos.

La competencia en el mercado eléctrico español responde, así pues, a un modelo de competencia oligopolística con un reducido número de agentes que participan en el mercado y se caracteriza por elevados niveles de concentración, aunque ésta ha ido decreciendo a medida que el proceso de liberalización ha permitido que nuevos agentes intervengan en el mercado. Así por ejemplo el índice de concentración $\mathrm{C}_{2}$ ha pasado de 0,70 en 2002 a 0,43 en $2008^{18}$. Sin embargo, la amenaza latente de que puedan ejercer abuso de posición dominante es aún elevada, y este hecho se puede ver agravado con la inclusión de los procesos de producción de energía basados en FER. La cuota de mercado de la generación mediante FER ha ido incrementándose a medida que ésta se ha ido transaccionando a través del mercado diario e intradiario. Esto supone que, tal y como se ha explicado anteriormente, la remuneración de la generación procedente de FER dependa de la fijación del precio en ambos mercados, y por lo tanto esté sujeta al comportamiento estratégico de las empresas que pueden ejercer posición de dominio. El Gráfico 1 muestra el porcentaje de capacidad y generación de electricidad mediante FER sobre el total para el período 2002-2008. El gráfico revela la tendencia creciente en la instalación y uso de FER.

En efecto, un generador que esté capacitado para introducir en la red una cantidad lo suficientemente grande de energía generada a partir de FER y reducir así su dependencia de los recursos fósiles, puede obligar a que el precio final sea más elevado ofreciendo las últimas unidades marginales de producción a precios elevados. Si dicha empresa no fuera pivotal, podría darse el caso de que no llegase a casar sus ofertas. Sin embargo, aunque no sea unilateralmente pivotal sí lo puede ser junto con otro generador. En ambos casos, al tratarse de una subasta a precio fijo, toda la energía generada recibe el pago de la última unidad marginal que se ha ofrecido a su cruce con la función de demanda, y que será fijada con la tecnología más cara (dado el orden meritocrático de entrada de las pujas de oferta). Así, la remuneración que recibe el generador en cada espacio horario por el total de la energía transaccionada es mayor cuanto mayor es el tramo a precio cero que es capaz de introducir y cuanto más cara es la unidad marginal de energía que marca el precio marginal del sistema. Nótese que cuanto menor es el tramo horizontal (ofrecido a precio cero)

${ }^{17}$ Es por ello que en los años 2007 y 2008 aparecen ceros en las casillas correspondientes.

${ }^{18}$ Véase Pérez Arriaga et al. (2005) y Fabra (2006) para un análisis de diferentes medidas del grado de concentración. 


\section{GRÁFICO 1}

\section{RATIO RENOVABLES / TOTAL POTENCIA INSTALADA Y GENERACIÓN}

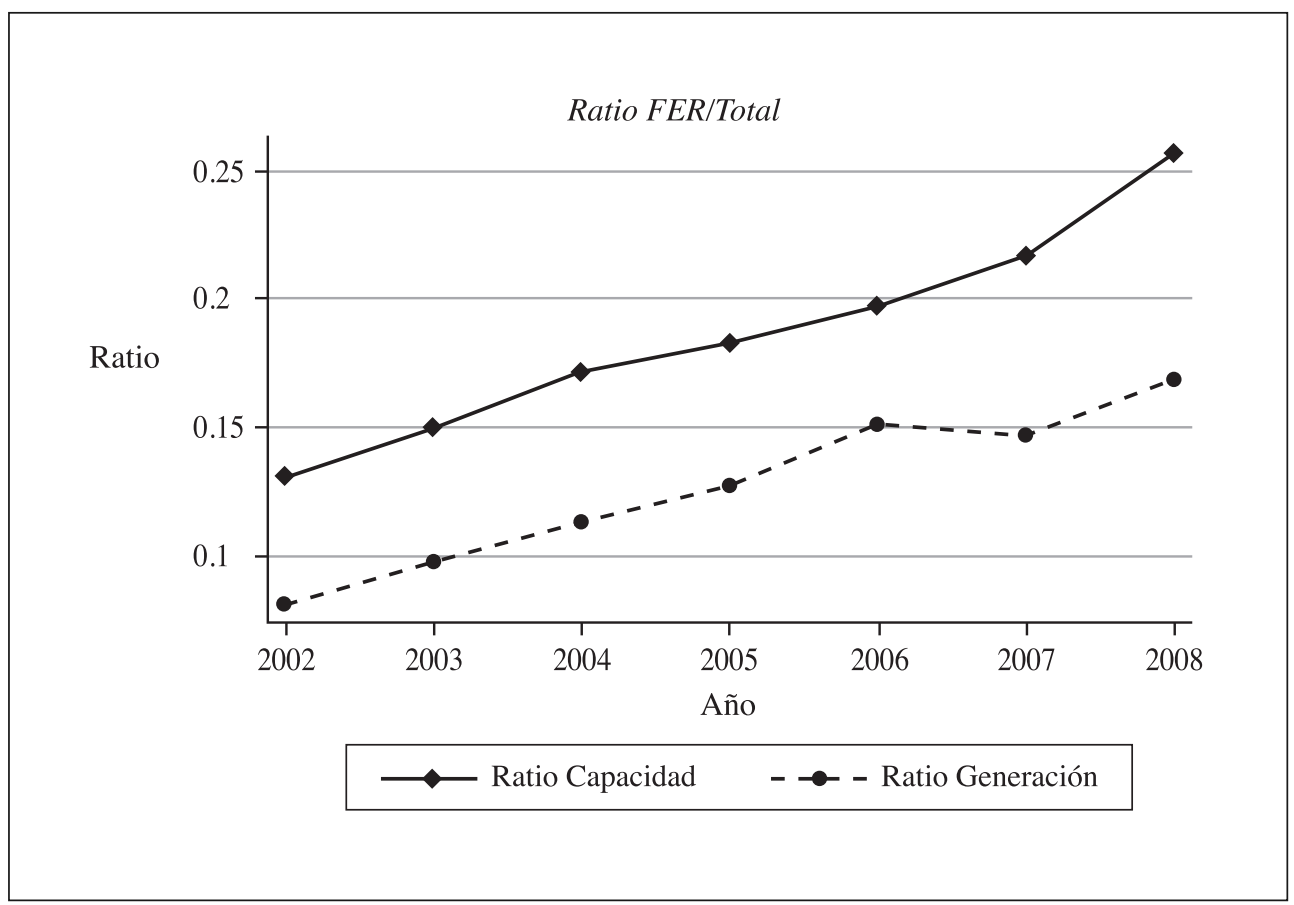

Fuente: OMEL, REE y construcción propia.

el incentivo a ofrecer la electricidad a un alto precio decrece ya que el porcentaje de oferta no casada es potencialmente mayor y el margen de beneficio se reduce e incluso puede ser cercano a cero. Hay que recordar que una función de oferta no refleja fielmente el coste marginal de generación.

Una virtud del actual marco regulatorio es que fomenta la inversión en generación procedente de FER y estimula el cierre de aquellas plantas de generación termoeléctricas más obsoletas y que no son necesarias para satisfacer el consumo en horas de demanda punta. El Cuadro 2 resume la potencia instalada por tecnología. Distinguimos dentro del régimen especial la generación procedente de fuentes renovables frente a la cogeneración. Además, por sus características y problemática peculiar la generación hidroeléctrica no se incluye dentro de las renovables. Dentro de la generación termoeléctrica distinguimos entre nuclear y convencional. Esta última agrupa plantas que utilizan como fuente de combustión carbón, fuel-gas y aquellas de ciclo combinado.

Se observa, al igual que en el Gráfico 1, el fuerte crecimiento que ha experimentado la generación procedente de FER. Así, mientras la tasa media acumulada de crecimiento de la energía producida a través de FER ha sido del 17\%, el crecimiento de 


\section{CUADRO 2}

POTENCIA INSTALADA POR TECNOLOGÍAS (GW)

\begin{tabular}{|c|c|c|c|c|c|c|}
\hline \multirow{2}{*}{ Año } & \multicolumn{3}{|c|}{ Régimen especial } & \multicolumn{2}{c|}{ Termoeléctrica } & \multirow{2}{*}{ Total } \\
\cline { 2 - 6 } & Renovables & Cogeneración & Hidroeléctrica & Nuclear & Convencional & \multirow{2}{*}{ Tonnnnn} \\
\hline 2002 & 7,8 & 5,6 & 16,6 & 7,8 & 21,9 & 59,6 \\
\hline 2003 & 9,4 & 5,6 & 16,6 & 7,8 & 22,9 & 62,3 \\
\hline 2004 & 11,8 & 5,7 & 16,6 & 7,8 & 26,7 & 68,8 \\
\hline 2005 & 13,5 & 5,7 & 16,6 & 7,8 & 30,3 & 74,1 \\
\hline 2006 & 15,7 & 5,8 & 16,6 & 7,7 & 33,6 & 79,5 \\
\hline 2007 & 18,8 & 6,0 & 16,6 & 7,7 & 37,5 & 86,6 \\
\hline 2008 & 23,5 & 6,1 & 16,6 & 7,7 & 37,8 & 91,8 \\
\hline
\end{tabular}

Fuente: OMEL, CNE y construcción propia.

la termoeléctrica convencional ha sido del $8 \%$, lo que ha permitido que la ratio de capacidad de renovables sobre el total pase de 0,13 en 2002 a 0,26. Esto permite a España colocarse a la cabeza de los países con mayor capacidad de generación en este tipo de tecnologías y cumplir con los objetivos de la Directiva 2001/71/CE.

En el Cuadro 3 presentamos un análisis similar que corrobora que la potencia instalada tiene una alta correlación con la generación efectiva de electricidad mediante FER. Se detalla a continuación la evolución de la generación por tecnología, siguiendo la misma clasificación.

\section{CUADRO 3}

\section{GENERACIÓN POR TECNOLOGÍA (GWH)}

\begin{tabular}{|c|c|c|c|c|c|c|c|}
\hline \multirow{2}{*}{ Año } & \multicolumn{3}{|c|}{ Régimen Especial } & \multicolumn{2}{|c|}{ Termoeléctrica } & \\
& Renovables & Cogeneración & Hidroeléctrica & Internacional & Nuclear & Convencional & \multirow{2}{*}{ Total } \\
\hline 2002 & 17.907 & 18.290 & 21.234 & 9.413 & 60.596 & 93.469 & 220.909 \\
\hline 2003 & 23.147 & 18.995 & 36.316 & 8.115 & 59.571 & 90.251 & 236.395 \\
\hline 2004 & 27.631 & 19.269 & 28.132 & 7.814 & 61.416 & 99.467 & 243.729 \\
\hline 2005 & 32.960 & 18.722 & 15.930 & 8.676 & 55.380 & 127.720 & 259.388 \\
\hline 2006 & 35.395 & 16.745 & 16.866 & 11.708 & 54.496 & 98.474 & 233.684 \\
\hline 2007 & 40.532 & 17.715 & 26.752 & 9.913 & 53.120 & 127.731 & 275.763 \\
\hline 2008 & 47.663 & 21.192 & 20.435 & 6.799 & 57.033 & 129.950 & 283.072 \\
\hline
\end{tabular}

Fuente: OMEL, CNE y construcción propia. 
En el mix de generación eléctrica la cuota de renovables ha pasado del 8,1\% en 2002 al 16,8\% en 2008, lo que supone además su máximo histórico. El RD 661/2007 ha tenido un efecto de estímulo también sobre la generación. Cuando se incluye la cogeneración, la proporción de electricidad procedente del régimen especial se incrementa del $15 \%$ en 2002 al 23\% de 2008. Es más, cuando se incluye la generación hidroeléctrica, el aumento es del $25 \%$ en 2002 al $31 \%$ en 2008. En consecuencia, España no está lejos de alcanzar el objetivo fijado por la Directiva 2001/77/CE ${ }^{19}$.

A pesar de que a nivel agregado el crecimiento en el uso de FER ha sido significativo, este no ha sido homogéneo para todas las empresas del sector. En el Cuadro 4 se resume la potencia instalada desagregada por generadores en el año 2006, anterior a la entrada en vigor del último gran paquete normativo en relación al régimen especial y en particular a las FER y el año 2008, donde se puede ver el cambio acaecido por efecto de la nueva normativa.

\section{CUADRO 4}

POTENCIA INSTALADA POR TECNOLOGÍA Y EMPRESA (GW)

\begin{tabular}{|c|l|r|r|r|r|r|r|r|r|}
\hline \multirow{4}{*}{ Año } & & EN & IB & UF & HC & GN & VI & Otras & Total \\
\hline \multirow{4}{*}{2006} & Renovables & 0.5 & 4.0 & 0.4 & 1.2 & 0.3 & 0 & 9.4 & $\mathbf{1 5 . 7}$ \\
\cline { 2 - 11 } & Cogeneración y otras & 0.3 & 0.4 & 0 & 0 & 0 & 0 & 5.1 & $\mathbf{5 . 8}$ \\
\cline { 2 - 11 } & Hidroeléctrica & 5.3 & 8.6 & 1.7 & 0.4 & 0 & 0.7 & 0 & $\mathbf{1 6 . 7}$ \\
\cline { 2 - 11 } & Termoeléctrica & 12.7 & 11.8 & 5.3 & 2.6 & 2.9 & 1.7 & 4.2 & $\mathbf{4 1 . 3}$ \\
\cline { 2 - 11 } & Total & $\mathbf{1 8 . 8}$ & $\mathbf{2 4 . 8}$ & $\mathbf{7 . 4}$ & $\mathbf{4 . 2}$ & $\mathbf{3 . 2}$ & $\mathbf{2 . 4}$ & $\mathbf{1 8 . 7}$ & $\mathbf{7 9 . 5}$ \\
\hline \multirow{5}{*}{2008} & Renovables & 2.9 & 5.5 & 1 & 1.4 & 0.4 & 0.4 & 11,9 & $\mathbf{2 3 , 5}$ \\
\cline { 2 - 11 } & Cogeneración y otras & 0.3 & 0.5 & 0.2 & 0 & 0 & 0 & 5.1 & $\mathbf{6 . 1}$ \\
\cline { 2 - 11 } & Hidroeléctrica & 5.3 & 8.6 & 1.7 & 0.4 & 0 & 0.7 & 0 & $\mathbf{1 6 . 7}$ \\
\cline { 2 - 11 } & Termoeléctrica & 13.1 & 11.9 & 7 & 2.8 & 3.7 & 1.7 & 5.3 & $\mathbf{4 4 . 6}$ \\
\cline { 2 - 11 } & Total & $\mathbf{2 1 . 6}$ & $\mathbf{2 6 . 5}$ & $\mathbf{9 . 9}$ & $\mathbf{4 . 6}$ & $\mathbf{4 . 1}$ & $\mathbf{2 . 8}$ & $\mathbf{2 2 . 3}$ & $\mathbf{9 1 . 8}$ \\
\hline
\end{tabular}

Fuente: REE, páginas web de las empresas y estimación propia.

19 En 2009 la producción eléctrica disminuyó un 5,1\%, hasta los 300.684 millones de Kw/h y paralelamente las emisiones de $\mathrm{CO}_{2}$ se contrajeron un $17 \%$. En cuanto a las fuentes de producción, destaca la caída del 10,5\% registrada en la nuclear y los descensos del 23,7\% y el 9,1\% en la producida con carbón y gas natural, respectivamente. En sentido contrario la energía generada a partir de FER se incrementó un 15,3\% junto con un incremento del 8,4\% de la hidroeléctrica. Así, en 2009 las FER produjeron el $18 \%$ del total de electricidad generada en España, el mismo porcentaje que la nuclear, con lo que las dos se colocan como segunda fuente de generación por detrás del gas natural, con un $36 \%$. La producción a partir de carbón generó un $13 \%$ de la electricidad total, las instalaciones hidroeléctricas un $9 \%$ y los productos petrolíferos el $6 \%$ restante. 
Como muestra la evidencia empírica, el mix de generación eléctrica en España se basa en un fuerte crecimiento de las FER. Este crecimiento viene principalmente de la mano de la energía eólica y, también dentro del Régimen especial, los ciclos combinados. Estos últimos se nutren del gas natural importado bien por gasoductos bien mediante la apertura de plantas de licuefacción. El impulso de las FER ha sido importante en todas las empresas aunque destaca Iberdrola sobre las demás (a través de su división Iberdrola Renovables) con un fuerte proceso de inversión y crecimiento en parques eólicos. Dicho proceso le ha llevado a desbancar a Endesa como empresa dominante por capacidad instalada en el sector en 2005. Más recientemente Viesgo, tras su fusión con EoN, está adoptando una política similar de expansión de las FER en su proceso de generación. En el Gráfico 2 presentamos la cobertura de la demanda para el año 2008 desagregada por fuentes de generación como porcentaje sobre el total. Se puede observar cómo la energía eólica constituye ya un porcentaje elevado con respecto al total. Junto con los recursos hidráulicos y el resto de renovables (fundamentalmente fotovoltaica y biomasa) constituyen el $22,5 \%$ de la demanda no siendo, sin embargo, las fuentes de energía que marcan los precios marginales. Esto último constituye un problema de incentivos muy complejo, ya que aquellos recursos más caros son los que marcan los precios al entrar en último lugar en el orden de casación.

\section{GRÁFICO 2}

\section{COBERTURA DE LA DEMANDA: 2008}

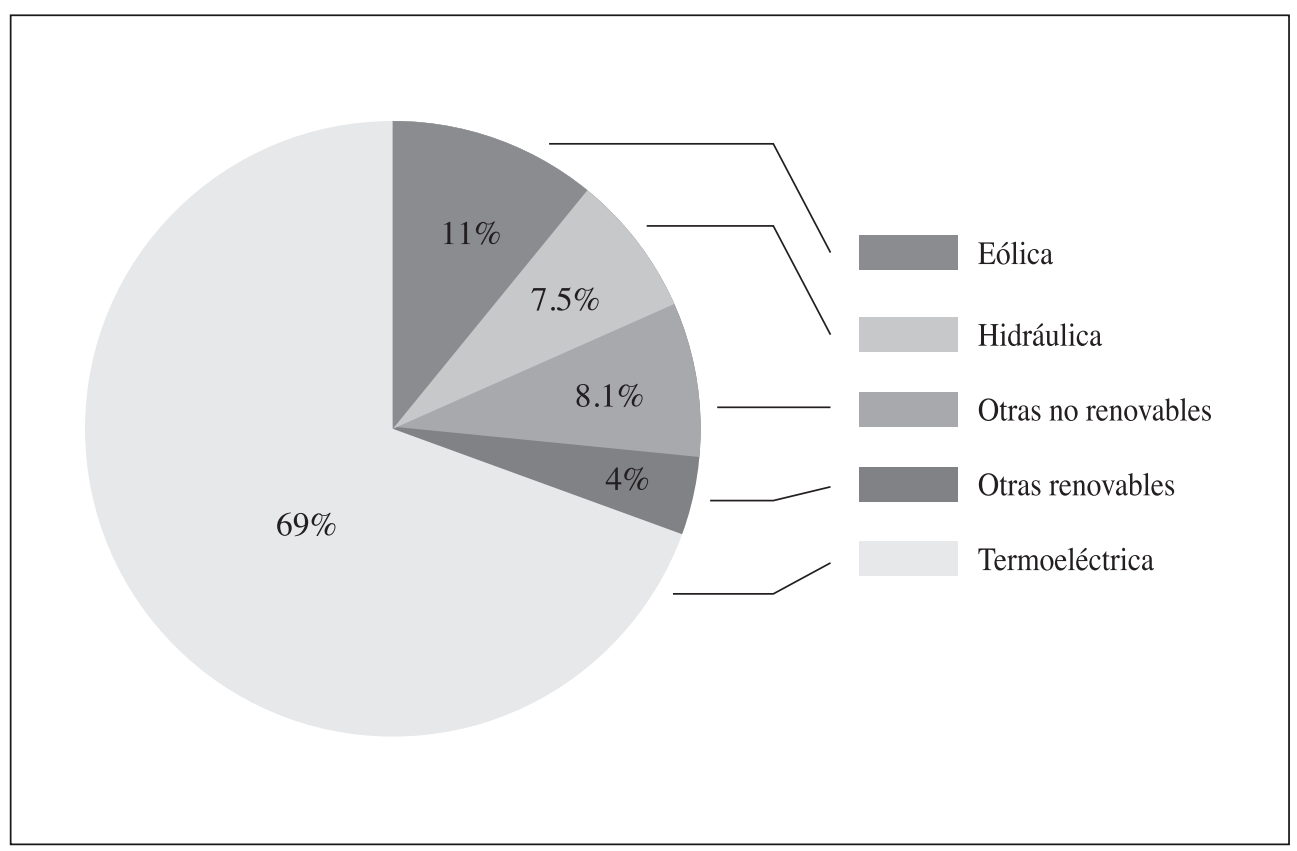




\section{Evaluación de los instrumentos de apoyo a las FER}

La Comunicación de la Comisión sobre el apoyo a la generación de electricidad a partir de FER [COM (2005) 627 final], evaluaba la aplicación de la Directiva 2001/77/CE en los distintos Estados miembros concluyendo, entre otras consideraciones, que los sistemas más eficaces en el caso de la energía eólica son actualmente los sistemas de primas de Alemania, España y Dinamarca. A continuación vamos a evaluar la eficiencia de los diferentes sistemas en función de 4 aspectos.

\section{a) Incentivos a la generación}

Los dos sistemas de incentivos presentan diferencias radicales en términos de rentabilidad futura. El sistema de primas en España ha servido para incentivar la generación eólica, tanto en términos de capacidad instalada como transaccionada (ver cuadros 2 y 3). Los beneficios generados por las inversiones en tecnologías que utilizan FER han sido elevados hasta ahora debido a los altos precios pagados a los generadores. Al no existir riesgo de mercado, la rentabilidad de las inversiones ha dependido sólo de la habilidad de los inversores para controlar los costes. Finalmente, los costes de transacción son menores que bajo otros sistemas más complejos a la hora de llevarlos a cabo. Por otra parte, los precios fijados a través de sistemas de pujas son más reducidos, debido a la presión de la competencia. Sin embargo el riesgo de las inversiones es mayor y así éstas son mucho más limitadas. Además, los márgenes de beneficio generados son mucho más reducidos que los márgenes generados con primas fijas o cuasi-fijas en el tiempo. La influencia del riesgo en los beneficios esperados es claramente una desventaja para los sistemas basados en pujas.

\section{b) Incentivos a la innovación}

El argumento principal para la utilización de primas es que ofrecen un alto nivel de certeza en la obtención de beneficios derivados de la inversión, tanto a las empresas generadoras como a los medianos y pequeños productores particulares (aversos al riesgo) garantizando un precio fijo para cada $\mathrm{kW} / \mathrm{h}$ de potencia casada y distribuida en red durante un cierto período de tiempo, generalmente dilatado. Como apuntamos previamente, esta certeza puede aplicarse en el corto o el medio plazo pero a largo plazo las primas pueden llegar a ser insostenibles debido a las ineficiencias en costes o bien porque no es compatible con un mercado liberalizado y competitivo y una política energética armonizada dentro de la UE.

El establecimiento de procesos de innovación dinámicos depende en buena parte de la curva de aprendizaje relativa a la difusión de las tecnologías, pero también de los procesos de $\mathrm{I}+\mathrm{D}+\mathrm{I}$ de los productores. Los sistemas de primas benefician tanto a los inversores como a los productores, pero si las primas no se ajustan a la vez que 
se incrementa el cambio tecnológico con las subsiguientes reducciones en costes el sistema deja de ser efectivo. En el caso de los procesos competitivos de pujas los generadores pueden repercutir los ahorros en costes a los consumidores finales.

\section{c) Eficiencia}

Debido a la certeza en la rentabilidad de las inversiones en tecnologías que utilizan FER ofrecida por el sistema de primas, éste ha sido muy eficaz en especial con la energía eólica en países como Alemania, Dinamarca o España. Su eficacia, sin embargo, depende en gran parte del nivel de las primas aplicadas a cada tecnología así como de otros factores como los costes de producción, la existencia de otros esquemas de promoción a los recursos renovables, procedimientos administrativos u otras características específicas a nivel regional o nacional. Además, aparte de la energía eólica, las primas han tenido un efecto mucho más reducido en la promoción de otras formas de producción (como la fotovoltaica, por ejemplo) ${ }^{20}$. En este sentido, la crítica principal con respeto a las primas es que han fallado en ser eficientes tanto en términos estáticos (esto es, en ser capaces de asegurar que la electricidad es generada y vendida minimizando costes) y en términos dinámicos (esto es, ser capaces de promover la innovación). Comparado con otros esquemas de promoción, las primas generalmente fallan en incentivar las reducciones en el precio mayorista para la electricidad generada a partir de FER. Ello puede ser debido a,

i) las primas son fijadas por la autoridad reguladora. Sin embargo esta autoridad carece, por lo general, de la información adecuada y actualizada en cuanto a los costes de producción mediante FER. Por ello, aunque las primas son fijadas de forma individual dependiendo de cada tecnología, en la práctica es muy difícil fijar una prima correcta y a la vez apropiada en cada caso (al no existir información completa sobre los costes de generación y otros costes asociados) y que además, pueda variar deforma correcta a lo largo del tiempo. Además, puede ser impopular y políticamente difícil reducir las primas a lo largo del tiempo debido a los intereses económicos de los grandes generadores.

ii) Como los sistemas de primas no están basados en la competencia directa entre los que utilizan fuentes renovables (además de otras) y entre estos y los que sólo utilizan combustibles fósiles para la producción de electricidad, el incentivo para generar procesos de innovación es menos acusado que en esquemas basados en la pura competencia.

${ }^{20}$ En el caso de la energía eólica se constata que el sistema de primas ha tenido un efecto positivo en la innovación (eficiencia dinámica). Una forma de cuantificar el proceso innovador en generación eólica es utilizando la potencia unitaria de los aerogeneradores. En 1995 se instalaban aerogeneradores de 0,3 MW mientras que en la actualidad se instalan aerogeneradores de $2 \mathrm{MW}$. 
En un estudio reciente Huber et al. (2001) demuestra que las primas son un instrumento eficiente en la promoción de las energías renovables sólo si se dan ciertas condiciones:

- La función de costes generada a partir de tecnologías basadas en recursos renovables tiene baja pendiente y es predecible con una alta probabilidad;

- Las primas decrecen con el horizonte temporal en línea con la función de aprendizaje esperada de los costes de inversión.

- El período de tiempo durante el cual un generador recibe la prima es limitado y conocido.

- Las primas deben decrecer al tiempo que el porcentaje de energía verde sobre el total se incrementa.

Aunque estas condiciones puedan parecer fáciles de cumplir son, en general, de difícil seguimiento, especialmente la primera. Para la mayoría de los recursos renovables el conocimiento exacto de la función de costes en un contexto dinámico es muy escaso. Además, la evidencia disponible sugiere que la función de costes en un determinado momento del tiempo tiene generalmente una pendiente pronunciada (modularidad, coste que se incrementa más que proporcionalmente al crecer el output), mientras que la función de costes dinámica tiende a decrecer con el tiempo debido al progreso tecnológico. Así pues, es difícil determinar las primas a lo largo del tiempo y entre tecnologías.

\section{d) Compatibilidad con el mercado y la competencia}

Otra de las críticas más importantes del sistema de primas es que implica una distorsión a la libre competencia en el contexto de un mercado liberalizado y único a nivel europeo. En particular, existen tres problemas,

i) Las primas no discurren por la misma senda de los precios fijados por la libre competencia entre generadores. Esto puede inducir a generar ineficiencias en la energía generada a partir de recursos renovables.

ii) Un sistema nacional de primas establece éstas para los generadores nacionales excluyendo del mismo a los productores extranjeros lo que puede generar conflictos de intereses en un contexto de mercado único de electricidad en el marco de la UE. La normativa europea explícitamente indica que no debe haber discriminación entre productores de distintos Estados miembros. Si existe discriminación entre generadores puede dar lugar a flujos financieros entre Estados miembros hacia aquellos que ofrecen primas más beneficiosas.

iii) Los generadores que están localizados en áreas más favorecidas para el uso y explotación de recursos renovables pueden ofrecer una mayor producción 
de electricidad y así recibir un volumen de primas mayor. Esto pone en clara desventaja a aquellos situados en áreas de bajo aprovechamiento, luego hay que diseñar mecanismos de compensación, lo que genera al menos un coste administrativo de seguimiento alto.

Algunos de los problemas planteados en torno a un sistema basado en primas son fácilmente solubles introduciendo primas que perciben todos los generadores de energía renovable por igual, incluyendo un mecanismo de compensación que cubra a todos los operadores de red dentro de la UE. Sin embargo, además de los costes que genera y las ineficiencias, un sistema así genera unos costes administrativos muy altos.

Por otra parte, un sistema de primas es muy simple de llevar a cabo desde un punto de vista administrativo. Sin embargo, son muy costosas en términos de recursos, tanto si los clientes deben de pagar un precio extra por utilizar electricidad generada mediante FER tanto si es el gobierno el que subvenciona las primas mediante el presupuesto. En España, el déficit de tarifa generado en el pasado pone un techo no lejano a los recursos disponibles para cubrir el sistema de primas, siendo por tanto la única vía el cargar un precio extra a los distribuidores y comercializadores que pasarán dicho coste a los consumidores finales. La medida es políticamente muy impopular ya que los consumidores no están acostumbrados a incrementos significativos en el precio de la electricidad. Una ventaja de los sistemas basados en pujas competitivas es que el nivel de los subsidios para la generación mediante FER es controlado por el regulador. A este respecto, los sistemas basados en cantidades han contribuido a que las autoridades puedan mantener el control del gasto a través de la revelación de la función de costes por parte de los productores. Se podría obtener un resultado similar con un sistema de primas si éste fuera menos rígido, es decir, ajustando las primas a la senda del progreso tecnológico a medida que las tecnologías sean progresivamente más competitivas.

Los modelos de competencia oligopolística nos permiten demostrar que los precios mayoristas tienden a disminuir cuanto mayor es la eficiencia productiva ya que los costes de producción descienden. Las plantas que generan electricidad a partir de fuentes no renovables, de origen no hidroeléctrico, operan con costes de producción más elevados, por lo tanto es menos competitiva que la generación térmica convencional. Por lo tanto, se precisa de un marco regulatorio que permita su crecimiento. Sin embargo, el nivel de la prima fijada por los reguladores puede de hecho favorecer un uso "excesivo" de dichas fuentes. Es posible demostrar que una subvención excesivamente alta puede falsear la competencia cuando la madurez técnica de las energías renovables es grande en comparación con los costes de las fuentes fósiles.

A nivel teórico, existe poco consenso en cuanto a los efectos sobre la competencia de la incorporación de FER ante diferentes marcos regulatorios. Es más, la literatura tanto teórica como empírica es aún escasa. Palmer y Burtraw (2005) han analizado el cambio climático y la política energética, encontrado que las políticas 
que buscan reducir las emisiones de gases de efecto invernadero procedentes del sector de la electricidad aumentan los costes económicos y precios de la electricidad. Por otra parte, Clemer et al. (1999) revelan que estas políticas efectivamente pueden resultar en precios más bajos para los consumidores. Ellos argumentan que la energía renovable adicional desplaza a la generación de turbinas de gas y la consiguiente disminución de la demanda reduce el precio del gas natural y, por último, el precio final de electricidad.

\section{Conclusiones y recomendaciones de política regulatoria}

A pesar del progreso conseguido en varias tecnologías que utilizan FER, existen otras que todavía no han alcanzado un nivel adecuado de desarrollo para competir con las fuentes de energía convencionales que utilizan combustibles fósiles. $\mathrm{Si}$ el progreso tecnológico se mantiene y las imperfecciones del mercado se corrigen ambas tecnologías podrían competir en el medio plazo por la producción de electricidad en red. Desde un punto de vista teórico, la solución eficiente para establecer competencia entre ambos tipos de tecnologías de generación es mediante una tasa o impuesto ambiental a aquellas tecnologías contaminantes y subvencionando aquellas que generan una energía limpia. Esta medida, sin embargo, debe estar acompañada con un incentivo a la innovación tecnológica y a cambios en el comportamiento del consumidor. En la práctica, un impuesto ambiental no es suficiente en sí mismo para estimular el proceso de innovación, por ello es necesario un sistema de primas e incentivos para precursar el uso de FER. Estas primas e incentivos están basados en precios, donde los generadores reciben una prima, o en cantidades, donde las autoridades públicas fijan un objetivo de generación que debe ser alcanzado por las empresas generadoras.

España ha hecho un esfuerzo significativo para cumplir los objetivos de la UE en el consumo de electricidad a partir de FER, tal y como se muestra en los Cuadros 2 y 3 sobre capacidad instalada y generación, respectivamente. La mejora tecnológica y el proceso de aprendizaje han hecho que en el medio plazo sea posible aumentar aún más la presencia de energía procedente de FER en el sistema eléctrico español, reducir los problemas de casación del operador del mercado y reducir la necesidad de generación por medio de combustibles fósiles. Para consolidar este objetivo es esencial ofrecer los incentivos adecuados a los agentes dentro de un marco normativo y regulatorio estable que permita internalizar los avances tecnológicos y crear incentivos para desarrollar nuevos proyectos.

En situaciones ideales, tanto un sistema basado en precios como basado en cantidades deben ser comparables y efectivos para conseguir un objetivo concreto en relación a los recursos renovables. Sin embargo, hay que tener en cuenta la incertidumbre asociada a su disponibilidad que depende en gran medida de condiciones climatológicas, por una parte, y del desarrollo tecnológico en el futuro cercano, por otra. Por ello, la eficiencia relativa de cada instrumento depende en gran medida de 
estas dos últimas cuestiones. En consecuencia, cuando evaluamos estas dos clases de instrumentos, hemos de utilizar diferentes criterios para evaluar su eficiencia a la hora de incentivar el uso de recursos renovables y el cambio tecnológico:

i) Control de costes. Un sistema basado en cantidades es más efectivo para controlar el coste en que incurre el estado al llevar a cabo políticas energéticas ya que mediante la asignación de cuotas para cada uno de los períodos es posible mantener el control directo sobre la capacidad instalada e indirecto sobre el coste marginal de producción. Un control similar puede conseguirse mediante los llamados certificados verdes. Sin embargo, bajo un sistema de primas, la producción de electricidad mediante FER no puede ser anticipada con precisión debido a la incertidumbre generada sobre los costes de producción. La única solución consistiría en ajustar los precios de acuerdo a la actuación de cada generador, pero esto sólo sería posible si todos ellos se enfrentasen a las mismas condiciones de producción (climáticas, restricciones técnicas, etc...). En la práctica, este tipo de control sería difícil de llevar a cabo por razones políticas e institucionales.

ii) Capacidad instalada. Un sistema basado en primas ha dado resultados mucho mejores que los sistemas basados en cantidades. En teoría, no debería haber tal diferencia, ya que se pueden establecer niveles de primas en las tarifas que proporcionen un mismo nivel de producción que un sistema basado en cantidades (dando lugar a una capacidad instalada comparable). La diferencia se explica por el mayor atractivo de un sistema de precios fijos, al garantizar a los generadores que los nuevos proyectos son inversiones sin riesgo bajo un sistema de incentivos estable, y con unos menores costes de transacción.

iii) Estímulo del cambio tecnológico. El incentivo para reducir costes es mucho más fuerte en un sistema de licitación pública, ya que los generadores que compiten en un mercado oligopolístico deben reflejar los costes más bajos posibles con el objetivo de conseguir subvenciones. En un sistema de primas hay, sin embargo, menos incentivos para reducir costes ya que disminuciones en costes no son automáticamente reflejadas en el sistema de incentivos. No obstante, es posible una reducción gradual de las primas en las tarifas que tengan en cuenta los avances en las tecnologías de energías renovables.

En términos generales, se puede afirmar que un sistema basado en primas puede ser efectivo como instrumento para promover la generación eléctrica mediante recursos renovables. En particular, en las primeras etapas de desarrollo de las diferentes fuentes (eólica y fotovoltaica, principalmente) a nivel nacional. A largo plazo, sin embargo, este sistema pude convertirse en un lastre para el mercado y la producción debido a la dificultad para su financiación, especialmente en etapas donde el porcentaje de energía verde es elevado sobre el total de la energía producida. Esta des- 
ventaja es más notoria si el sistema de primas es fijo, ya que distorsiona la formación de precios competitiva y, en consecuencia, es incompatible con la creación de un mercado liberalizado y único a nivel europeo. En el largo plazo el mejor camino para promover el uso de recursos renovables en la generación eléctrica dentro de un mercado liberalizado a nivel europeo es internalizar los costes asociados al uso de los recursos no renovables, por ejemplo vía tasas o impuestos, o bien introducir un sistema harmonizado de certificados verdes negociables en los que se incluyan los beneficios ambientales de los recuros renovables frente a los no renovables evaluados económicamente. Sin embargo, debe existir un período de tiempo que dependerá de la maduración del proceso de los recuros renovables para ser plenamente competitivos donde deberían combinarse ambos instrumentos, teniendo en cuenta que cuanto más incipiente es el proceso de uso de FER deberían utilizarse más profusamente un sistema de primas.

El grado de cumplimiento de objetivos tales como la reducción del calentamiento global y un suministro energético seguro y eficiente, adoptados en diciembre de 2008 por los líderes de la UE, depende en buena medida de la evaluación de políticas y mecanismos que favorezcan el uso de FER en un horizonte cercano. En el caso español, la reciente Ley de Economía Sostenible establece explícitamente en el Artículo 3 del Título Preliminar la promoción de las energías limpias y la reducción de emisiones. En éste sentido, el Artículo 98 reconoce explícitamente la necesidad de internalizar todos los costes y beneficios provocados por el uso de FER.

\section{Referencias bibliográficas}

[1] CIARRETA, A. y GUTIÉRREZ-HITA, C. (2009): "Entering renewable energy sources in the Spanish electricity market: The effects of regulatory reforms", IAEE Newsletters, Third Quarter, pp. 21-23.

[2] CLEMER, S.; NOGEE, A. y BROWER, M. (1999): "A powerful opportunity: Making renewable electricity the standard". Cambridge, MA, Union of Concern Scientists (UCS). January.

[3] COM (2005) 627 final (7/12/2005).

[4] DIRECTIVA 1996/61/CE.

[5] DIRECTIVA 1996/92/CE.

[6] DIRECTIVA 2001/71/CE.

[7] DIRECTIVA 2001/77/CE.

[8] DIRECTIVA 2003/54/CE.

[9] DIRECTIVA 2003/87/CE.

[10] FABRA, N. (2006): "El funcionamiento del mercado eléctrico español bajo la Ley del Sector Eléctrico", en "Del monopolio al mercado. La Comisión Nacional de la Energía: diez años en perspectiva". Editorial Thomson, Civitas.

[11] HUBER, C.; HAAS, R. y WOHLGEMUTH, N. (2001): "Financial incentives to promote renewable energy systems in European electricity markets - A survey". International Journal of Global Energy Issues, Vol. 15, pp. 5-24. 
[12] JAMASB, T. y POLLITT, M. (2003): “International benchmarking and regulation: an application to European electricity distribution utilities. Energy Policy, 31, pp. 1609-1622.

[13] LEY 82/1980.

[14] LEY 54/1997, del sector eléctrico.

[15] LEY 40/1994.

[16] LEY 17/2007.

[17] OMEL, http://www.omel.es.

[18] ORDEN ITC/3801/2008, (26/12/2008).

[19] PALMER, K. y BURTRAW, D. (2005): “Cost-effectiveness of renewable electricity policies". Energy Economics 24, pp. 557-576.

[20] PÉREZ ARRIAGA, J. I.; BATTLE, C.; VÁZQUES, C.; RIVIER, M. y RODILLA, P. (2005): "Libro Blanco sobre la reforma del marco regulatorio de la generación eléctrica en España. ISBN 978-84-4785-6.

[21] RD 2366/1994, BOE 313, 31/12/1994.

[22] RD 2017/1997, BOE 310, 27/12/1997.

[23] RD 2818/1998, BOE 312, 30/12/1998.

[24] RD 841/2002, BOE 210, 02/09/2002.

[25] RD 435/2004, BOE 73, 25/03/2004.

[26] RD 436/2004, BOE 75, 27/03/2004.

[27] RD 661/2007, BOE 178, 26/07/2007.

[28] RD 1578/2008, BOE 234, 27/09/2008.

[29] RD-LEY 6/2000, BOE 151, 24/06/2000.

[30] RD-LEY 6/2009, BOE 111, 07/05/2009.

[31] RES-E White Paper, COM (1997) 599 final (26/11/1997). 
